\section{Band-reject ultra-wideband monopole antenna using patch loading}

\section{Koohestani, N. Pires, A.K. Skrivervik and A.A. Moreira}

Proposed is a new simple technique for achieving band rejection characteristics in ultra-wideband monopole antennas. Two band notches were added to an existing UWB antenna by gluing a padding patch printed on a small single-layer piece of commercial substrate. The design of the new proposed structure has a controllable rejection in the $5 \mathrm{GHz}$ WLAN and $8 \mathrm{GHz}$ ITU frequencies while maintaining a wideband performance from $2.9 \mathrm{GHz}$ to more than $12 \mathrm{GHz}$ based on VSWR $\leq 2$. A good agreement between measurement and simulation was achieved. Measurements confirm that the technique introduces negligible antenna radiation pattern distortion except at the notched frequencies.

Introduction: The existence of a large number of wireless applications leads to a very heavy congestion in the available radio-frequency spectrum. This results in a significant interference among different services, which can cause severe system performance degradation. Specifically, ultra-wideband (UWB) applications share a part of the spectrum with the narrowband, and higher powered, IEEE 802.11 family of Wireless Local Area Network (WLAN) $(5.15-5.35 \mathrm{GHz}, 5.725-5.825 \mathrm{GHz})$ used in America and the International Telecommunication Union (ITU) band (8.025-8.4 GHz) used in Europe. This fact fostered some interest in the wireless communications community for ways to mitigate possible interferences. One solution is to electronically or digitally filter the problematic bands, with the undesirable side effect of increasing the system complexity and/or weight. Another alternative, discussed here, is to design the UWB antenna with band rejection characteristics.

Many techniques have been presented to introduce notch bands for reducing narrowband interference in UWB planar monopole antennas [1-6]. The most commonly used approaches are inserting a quarter or half of the guided wavelength slit on the antenna radiation elements, such as L-shaped [1], T-shaped [2], C-shaped [3], and U-shaped [4] slots. Other techniques of rejecting the undesired frequencies are the addition of either a split ring resonator [5] or a multi-resonator load [6] in the antenna structure.

This Letter proposes a new simple approach to obtain controllable frequency notches for both WLAN and ITU bands using a variation of the dielectric loading sandwich technique [7]. It consists of gluing a padding patch printed on a single-layer substrate on the radiating element of the antenna. Simulations and measurements have been conducted to confirm the adequacy of the new design.
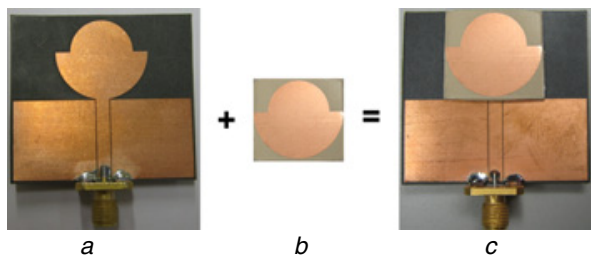

Fig. 1 Picture of UWB monopole antenna [9], proposed padding patch, and antenna with glued patch

$a$ UWB monopole antenna [9]

$b$ Proposed padding patch

$c$ Antenna with glued patch

Antenna structure and design: Fig. 1 shows the configuration of the proposed antenna and the padding patch. The radiator, shown in Fig. $1 a$, is a highly efficient (over 96\%) [8] coplanar-fed planar UWB monopole antenna (size $\left.44 \times 38 \mathrm{~mm}^{2}\right)$. It comprises two semicircles with different radii. The antenna was fabricated on a Rogers RT/ Duroid 5880 substrate with thickness of $1.57 \mathrm{~mm}$, relative permittivity of 2.2 and a loss tangent of 0.0009 . The backside of the substrate is devoid of any metallisation. The detailed design of the antenna has been presented in [9]. As it can be seen in Fig. 1c, a small rectangular padding, shown in Fig. $1 b$, with a similar etched conductor patch was glued on top of the antenna. Photographic aerosol glue was used to fix the padding in place. In this study, $R$ and $r$ represent the radius of the larger and smaller semicircles etched on the padding. A low loss substrate was chosen for the padding in order not to impact the antenna efficiency [10]. The pad was fabricated on a Rogers RO 3003 with $1.52 \mathrm{~mm}$ thickness, relative permittivity of 3 and a loss tangent of 0.001 . It should be noted that a thicker substrate causes the notch frequency bandwidth to increase, but lowers the rejection level of the notches. The width and length of the padding patch are 19 and $22 \mathrm{~mm}$, respectively.

Each notch frequency can be adjusted by keeping one semicircle radius fixed while changing the other. This can be explained by the fact that these semicircles are resonant and create large currents that cause destructive interference with the excitation, making the antenna non-responsive to the corresponding frequencies. The semicircle radil mainly determine the centre frequency of the notch band. This is apparent in Figs. 2 and 3, which show the simulation results for different radii values. The amount of the frequency shift of the notch frequencies against different radii with reference to $r=7.5 \mathrm{~mm}$ and $R=11 \mathrm{~mm}$ are displayed in Table 1 . The patch loading technique is also effective when only a single notch is intended. This can be achieved by cutting a slot in the padding patch of approximately half the guided wavelength of the required notch frequency, as implemented in [3]. This was confirmed by HFSS simulations for creating a single notch between 3.4 and $3.6 \mathrm{GHz}$, a band dedicated to worldwide interoperability for microwave access (WiMAX).

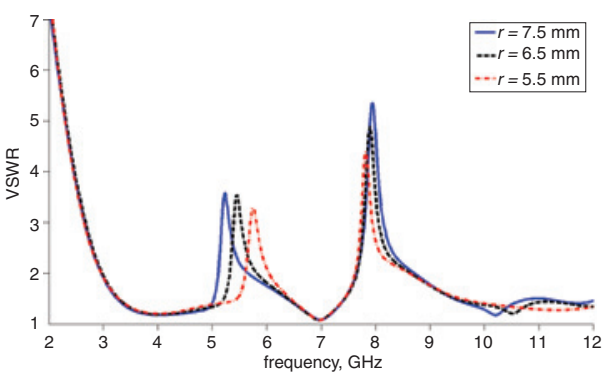

Fig. 2 Simulated VSWR for three values of antenna parameter $r$

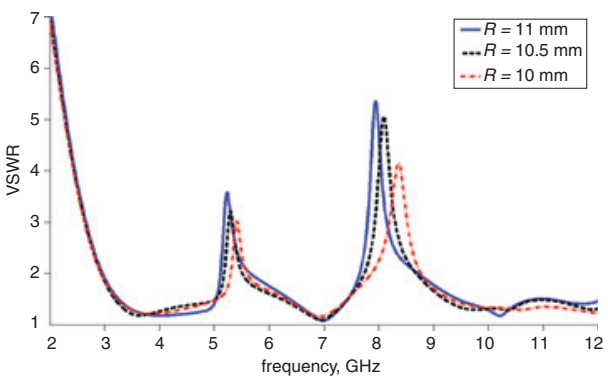

Fig. 3 Simulated VSWR for three values of antenna parameter $R$

Table 1: Shift in notch frequencies (\%) against different radii relative to notch frequencies obtained with $r=7.5 \mathrm{~mm}$ and $R=11 \mathrm{~mm}$ (U: upward, D: downward)

\begin{tabular}{|c|c|c|}
\hline Semicircles radii & Lower band & Upper band \\
\hline$r=5.5 \mathrm{~mm}, R=11 \mathrm{~mm}$ & $9.95 \%(\mathrm{U})$ & $1.51 \%(\mathrm{D})$ \\
\hline$r=6.5 \mathrm{~mm}, R=11 \mathrm{~mm}$ & $4.2 \%(\mathrm{U})$ & $0.5 \%(\mathrm{D})$ \\
\hline$r=7.5 \mathrm{~mm}, R=10.5 \mathrm{~mm}$ & $1.14 \%(\mathrm{U})$ & $2.01 \%(\mathrm{U})$ \\
\hline$r=7.5 \mathrm{~mm}, R=10 \mathrm{~mm}$ & $3.44 \%(\mathrm{U})$ & $5.29 \%(\mathrm{U})$ \\
\hline
\end{tabular}

Experimental results: The antenna performance in terms of VSWR, radiation pattern and gain response, has been studied. The antenna VSWR was simulated using Ansoft's High Frequency Structure Simulator ( $\mathrm{HFSS}^{\mathrm{TM}}$ ) and measured between 2 and $12 \mathrm{GHz}$ using an Agilent E8361 vector network analyser. Fig. 4 shows the measured and simulated VSWR for the proposed antenna with and without patch padding. The measurements confirm that the unpadded antenna fully covers the FCC UWB band. The patch padding introduces a stop-band in 5.35-5.8 and 7.98-8.85 GHz while maintaining the wideband performance from 2.9 to more than $12 \mathrm{GHz}$ with VSWR $\leq 2$. The shift towards lower frequencies for the padded antenna response is due to the dielectric loading effects of the padding patch substrate $\left(\epsilon_{r}=3\right)$ [10]. A reasonably good agreement between simulated and measured VSWR has been achieved. The observed discrepancies may be due to fabrication imperfections, especially alignment problems when gluing the patch padding. The antenna radiation patterns for the co-polar components in the H-plane ( $y-z$ plane) have been measured in an anechoic 
chamber. It should be noted that the $|\theta|>120$ region is omitted because of blockage by the antenna supporting tower. Measured results at frequencies out of the notch bands confirm the omnidirectional properties of the antenna, both with and without padding, showing that the presented technique does not significantly distort the radiation pattern. However, at notched frequencies, as expected, the antenna radiation patterns distort. Fig. 5 illustrates the measured radiation patterns normalised to their maximum values for the E-field co-polar component at notched bands central frequencies, 5.45 and $8.15 \mathrm{GHz}$. It can be seen that applying the patch causes antenna radiation pattern distortion at notched bands.

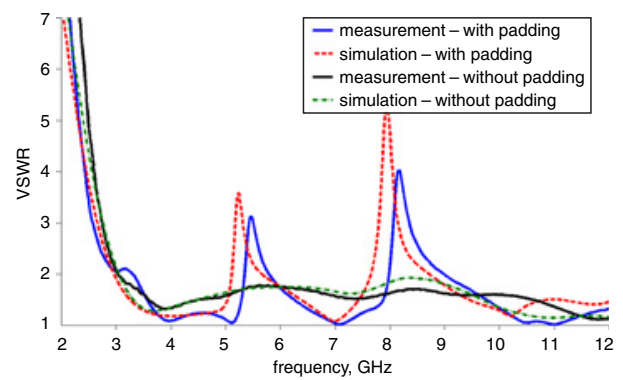

Fig. 4 Simulation and measurement results of padding patch effect showing added notch frequencies

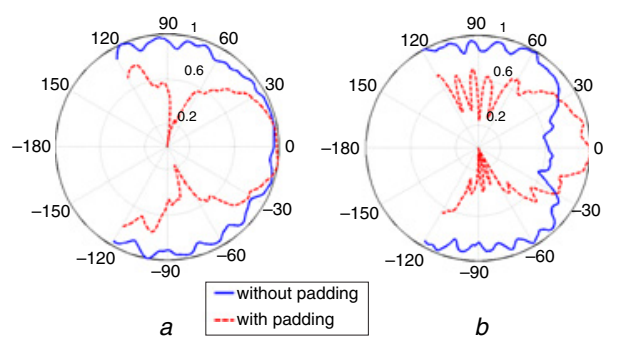

Fig. 5 Normalised radiation pattern for E-field co-polar component at notched frequencies

a $5.45 \mathrm{GHz}$

b $8.15 \mathrm{GHz}$

Conclusion: A small padding patch printed on a single-layer commercial substrate has been designed and used to introduce desired frequency notches in a printed UWB monopole antenna. It was confirmed through VSWR measurements that the designed structure has a rejection behaviour at the WLAN and ITU frequency bands. By varying the semicircles radii on the proposed padding, the notch frequencies can be easily adjusted. Measurements of the antenna radiation pattern have confirmed that applying the padding does not cause radiation pattern distortions. Total gain measurements also confirmed the intended behaviour of the padding in obtaining notched bands. Thus, it can be concluded that the proposed technique is suitable for creating an UWB antenna with narrow frequency-notched characteristics.
Acknowledgments: This work was supported by the Portuguese Fundação para a Ciência e a Tecnologia (FCT) project PEst-OE/EEI/ LA0008/2011. The work of N. Pires is supported by the FCT grant SFRH/BD/51066/2010 and M. Koohestani by the FCT grant SFRH/ $\mathrm{BD} / 51445 / 2011$.

(C) The Institution of Engineering and Technology 2012 24 May 2012

doi: $10.1049 /$ el.2012.1771

One or more of the Figures in this Letter are available in colour online. M. Koohestani, N. Pires and A.A. Moreira (Instituto de Telecomunicações, Instituto Superior Técnico, Universidade Técnica de Lisboa, Lisbon, Portugal)

E-mail: koohestani.mohsen@ist.utl.pt

A.K. Skrivervik (Laboratoire d'Electromagnétisme et d'Acoustique, École Polytechnique Fédérale de Lausanne, Lausanne, Switzerland)

M. Koohestani and N. Pires: Also with Laboratoire d'Electromagnétisme et d'Acoustique, École Polytechnique Fédérale de Lausanne, Lausanne, Switzerland

\section{References}

1 Choi, W., Chung, K., Chung, J., and Choi, J.: 'Compact ultra-wideband printed antenna with band-rejection characteristics', Electron. Lett., 2005, 41, (18)

2 Hong, C.Y., Ling, C.W., Tarnand, I.Y., and Chung, S.J.: 'Design of a planar ultrawideband antenna with a new band-notch structure', IEEE Trans. Antennas Propag., 2007, 55, (12)

3 Chu, Q.-X., and Yang, Y.-Y.: ' $3.5 / 5.5 \mathrm{GHz}$ dual band-notch ultrawideband antenna', Electron. Lett., 2008, 44, (3)

4 Ojaroudi, M., Ghanbari, G., Ojaroudi, N., and Ghobadi, C.: 'Small square monopole antenna for UWB applications with variable frequency band-notch function', IEEE Antennas Wirel. Propag. Lett., 2009,8

5 Kim, J., Cho, C.S., and Lee, J.W.: ' $5.2 \mathrm{GHz}$ notched ultra-wideband antenna using slot-type SRR', Electron. Lett., 2006, 42, (6)

6 Ma, T.G., Hua, R.C., and Chou, Ch.F.: 'Design of a multi resonator loaded band-rejected ultrawideband planar monopole antenna with controllable notched bandwidth', IEEE Trans. Antennas Propag., 2008, 56, (9)

7 Moreira, A., Pires, N., Serro, N., and Santos, R.: 'Ultra wideband dielectric sandwich loaded antennas'. Proc. of European Conf. on Antennas and Propagation, Berlin, Germany, 2009

8 Pires, N., Mendes, C., Koohestani, M., Skrivervik, A.K., and Moreira, A.A.: 'Radiation efficiency of a coplanar-fed ultra-wideband antenna'. IEEE AP-S/USNC-URSI, Chicago, IL, USA, 2012

9 Koohestani, M., Pires, N., Skrivervik, A.K., and Moreira, A.A. 'Influence of the human body on a new coplanar-fed ultra-wideband antenna'. Proc. of European Conf. Antennas and Propagation, Prague, Czech Republic, 2012

10 Pires, N., Letizia, M., Boyes, S., Lu, Y., Huang, Y., Skrivervik, A.K., and Moreira, A.: 'Improved design of an ultra wideband universal serial bus device mounted antenna based on comparative radiation efficiency measurements'. Proc. of European Conf. on Antennas and Propagation, Rome, Italy, April 2011 